\title{
Multimodality treatment of unresectable stage III non-small cell lung cancer: Interim analysis of a phase II trial with preoperative gemcitabine and concurrent radiotherapy
}

Domenico Galetta, MD a Alfredo Cesario, MD ${ }^{\mathrm{b}, \mathrm{g}}$ Stefano Margaritora, MD ${ }^{\mathrm{b}}$ Venanzio Porziella, $\mathrm{MD}^{\mathrm{b}}$ Alessio Piraino, MD, ${ }^{\mathrm{b}}$ Rolando M. D'Angelillo, MD, ${ }^{\mathrm{c}}$ Maria A. Gambacorta, MD, ${ }^{\mathrm{c}}$ Sara Ramella, MD, ${ }^{\mathrm{c}}$ Lucio Trodella, MD, ${ }^{\mathrm{c}}$ Salvatore Valente, ${ }^{d}$ Giuseppe M. Corbo, ${ }^{\mathrm{d}}$ Giuseppe Macis, ${ }^{\mathrm{e}}$ Antonino Mulè, ${ }^{\mathrm{f}}$ Vittorio Cardaci, MD, ${ }^{\mathrm{h}}$ Silvia Sterzi, MD, Pierluigi Granone, MD, and Patrizia Russo, $\mathrm{PhD}^{\mathrm{b}}$

Supplemental material is available online.
From the Department of Thoracic Surgery, European Institute of Oncology, Milan $;$; the Division of General Thoracic Surgery, ${ }^{\mathrm{b}}$ the Department of Radiotherapy, ${ }^{\mathrm{c}}$ the Division of Respiratory Physiology, ${ }^{\mathrm{d}}$ the Department of Radiology, ${ }^{e}$ and the Department of Pathology, ${ }^{\mathrm{f}}$ Catholic University, Rome; the Clinical, Respiratory Pathology Translational Laboratory ${ }^{\mathrm{g}}$ and the Department of Pulmonary Rehabilitation, ${ }^{\mathrm{h}}$ San Raffaele, IRCCS, Rome; and the Rehabilitation Service, University Campus Bio-Medico, Rome, Italy. ${ }^{i}$

Read at the Thirtieth Annual Meeting of the Western Thoracic Surgical Association, Maui, Hawaii, June 23-26, 2004.

Received for publication June 19, 2005; revisions received July 2, 2005; accepted for publication July 11, 2005 .

Address for reprints: Alfredo Cesario, MD, Division of General Thoracic Surgery, Catholic University, Largo A. Gemelli, 8, 00168 Rome, Italy (E-mail: alfcesario@rm. unicatt.it)

J Thorac Cardiovasc Surg 2006;131:314-21

$0022-5223 / \$ 32.00$

Copyright (๑) 2006 by The American Association for Thoracic Surgery

doi:10.1016/j.jtcvs.2005.07.044
Objective: We report the preliminary results of a phase II trial undertaken to determine the feasibility and efficacy of gemcitabine and concurrent radiotherapy in patients with inoperable stage III non-small cell lung cancer.

Methods: Between February 2001 and June 2003, a total of 46 patients (37 male and 9 female, median age 64 years) with clinical stage III non-small cell lung cancer (41 cIIIA and 5 cIIIB) were enrolled in a combined chemoradiation protocol with gemcitabine as the chemotherapeutic agent. Gemcitabine $\left(350 \mathrm{mg} / \mathrm{m}^{2}\right)$ was administered weekly for 5 consecutive weeks as a 30-minute intravenous infusion before radiotherapy (total dose $50.4 \mathrm{~Gy}, 1.8 \mathrm{~Gy} / \mathrm{d}$ ). Toxicity was routinely assessed. Those patients with disease judged to be resectable at restaging underwent surgery.

Results: Toxicity was moderate, with the exception of 1 grade 3 thrombocytopenia. All but 5 patients were available for restaging. No complete responses were observed. Thirty-four patients $(82.9 \%)$ had partial responses, $5(12.2 \%)$ had stable disease, and 2 (4.9\%) had progressive disease. Twenty-nine of 46 patients $(63 \%, 27$ cIIIA and 2 cIIIB) underwent surgery. Radical resection was possible in all cases. Surgery included 17 lobectomies, 4 bilobectomies, and 8 pneumonectomies. There were no deaths. Morbidity was $13.8 \%$ (4/29). Pathologic downstaging to stage 0 or I was observed in 18 patients $(39 \%, 18 / 46)$. After a median follow-up of 13 months (range 2-28 months), 24 of the patients who had undergone operation (86.2\%) were alive, with a median disease-free survival of 16 months. Overall 2-year survival was $66.1 \%$, with a significant difference between resected and unresected disease $(82 \%$ vs $36 \%, P=.0002$ ).

Conclusion: The results of this induction trial confirm the feasibility and the efficacy of gemcitabine with concurrent radiotherapy.

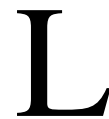
ung cancer remains the number one cancerous cause of death among both men and women in developed countries. It was estimated that during 2004 in the United States there would be about 173,770 new cases of lung cancer, with an estimated 160,440 deaths. ${ }^{1}$ Despite years of research, the prognosis for patients with lung cancer remains dismal, with a 5-year survival for all stages of $15 \%$. Surgery remains the mainstay of therapy for early-stage lung cancer, achieving 5-year survivals of 70\% for patients with stage I disease and 50\% for those with stage II. $^{2}$ Unfortunately, most patients with lung cancer are first seen with advanced (stage III) disease and are therefore less likely to benefit from surgery. Nor will they 


$$
\begin{aligned}
& \text { Abbreviations and Acronyms } \\
& \begin{aligned}
\mathrm{CT} & =\text { computed tomography } \\
\mathrm{NSCLC} & =\text { non-small cell lung cancer } \\
\mathrm{PD} & =\text { progressive disease } \\
\mathrm{PR} & =\text { partial response } \\
\mathrm{SD} & =\text { stable disease }
\end{aligned}
\end{aligned}
$$

benefit from any single-modality treatment-based approaches (either radiotherapy or chemotherapy).

The treatment of stage III non-small cell lung cancer (NSCLC) still remains challenging, with only complex multimodality treatments playing a role in improving results. Even if chemoradiotherapy is generally considered as the standard of care for locally advanced disease, ${ }^{3}$ strategies including neoadjuvant treatment followed by surgery have shown good results, motivating researchers to explore this approach. $^{4}$

There is a growing body of data regarding the multimodality neoadjuvant approach to locally advanced NSCLC. ${ }^{5-7}$ Although definitive conclusions cannot yet be drawn, several phase II trials and some published randomized studies $^{8-10}$ suggest that induction therapy is feasible, has insignificant toxicity, and may improve long-term survival. Most agents have been chosen for combination with radiotherapy on the basis of their known clinical activity in a particular disease site although none of them has been clearly superior. For example, agents such as cisplatin, 5-fluorouracil, and carboplatin have been largely used in chemoradiation regimens.

Recently, the introduction of such new agents as gemcitabine, vinorelbine, and taxanes for the treatment of NSCLC has generated great interest in the identification of more active and better tolerated combinations. In phase II studies, the gemcitabine-cisplatin combination has exhibited a favorable and safe profile. ${ }^{11-13}$ Gemcitabine $\left(2^{\prime}, 2^{\prime}\right.$ difluoro- $2^{\prime}$ deoxycytosine) is a cytosine analog with a modification in the 2 position of the sugar ring. It is one of the most extensively evaluated newer cytotoxic agents, characterized by favorable toxicity profiles and better tolerated than platinum-based regimens, ${ }^{14}$ with clinical activity in solid tumors including NSCLC through a broad spectrum of activity. Recent studies have demonstrated that by altering deoxynucleoside triphosphate pools ${ }^{15-17}$ gemcitabine acts as a potent radiosensitizer in several different solid tumor cell lines in vitro-including lung cancer-at noncytotoxic concentrations. Although the mechanism of sensitization is not yet clear, evidence suggests that gemcitabine lowers the threshold for radiation-induced apoptosis. ${ }^{17}$ In an in vitro study, Shewach and Lawrence ${ }^{15}$ demonstrated that shortterm (2-hour) exposure to gemcitabine was sufficient to produce radiosensitization when cells were irradiated after 24 or 48 hours, presumably because of the prolonged cellular effects mediated by lengthy retention of the triphosphate form. This suggests that once- or twice-weekly administration of gemcitabine could effectively sensitize tumor cells during a weekly course of radiotherapy. In a recent phase I and II trial with induction carboplatin and gemcitabine followed by concurrent chemoradiotherapy with vinorelbine and paclitaxel, followed by surgery, Argiris and colleagues ${ }^{18}$ reported good results in terms of toxicity, objective response to the treatment, and survival.

In a previous phase I controlled trial, we tested the use of gemcitabine as radiosensitizer administered concurrently with radiotherapy in the treatment of patients with unresectable locally advanced NSCLC, finding the maximum tolerable dose to be $350 \mathrm{mg} / \mathrm{m}^{2}$ weekly for 5 weeks. ${ }^{19}$ On the basis of this experience, we planned a prospective trial for patients with unresectable stage IIIA and IIIB NSCLC of a multiple modality therapy combining gemcitabine with concurrent radiation therapy and surgery. The objective of this study was to assess this new treatment in terms of feasibility, toxicity, tumor response, survival, and tumor recurrence.

\section{Patients and Methods}

This single-center, phase II, ongoing trial started in February 2001 at Policlinico Gemelli Hospital, Catholic University in Rome. Patient accrual terminated in June 2003. The protocol was approved by the local institutional review committee. All patients signed an informed consent form before being admitted to the study.

\section{Eligibility}

Eligibility criteria included patients 18 to 75 years old with a performance status of 0 or 1 on the Eastern Cooperative Oncology Group scale. Patients also must have had pathologically or cytologically confirmed locally advanced NSCLC judged inoperable or unresectable (stage IIIa or IIIb), with no pulmonary or cardiovascular contraindications to chemoradiotherapy and surgery. Other required criteria were as follows: leukocyte count greater than 4000 cells $/ \mu \mathrm{L}$, platelet count greater than 100,000 cells $/ \mu \mathrm{L}$, hemoglobin level greater than $10 \mathrm{~g} / \mathrm{dL}$, serum bilirubin level not more than 1.5 times the upper limit of normal, serum alanine aminotransferase and aspartate aminotransferase levels not more than 2.5 times the upper limit of normal, normal creatinine level, and creatinine clearance level greater than $60 \mathrm{~mL} / \mathrm{min}$. Exclusion criteria included previous oncologic treatment and history of another malignancy.

\section{Diagnostic Procedures}

Initial staging evaluation included patient history and physical examination with evaluation of performance status, complete blood cell count and hematochemical testing, electrocardiogram, bronchoscopy, pulmonary function test, arterial blood gas analysis, and perfusion nuclide scintigraphy. Chest radiography and computed tomography (CT) of the chest, abdomen, and brain, as well as bone scintigraphy, were performed to ensure the absence of metastatic dissemination. NSCLC diagnosis was obtained by fineneedle aspiration biopsy, endobronchial biopsy, or bronchoscopic 
brushing or washing. Surgical staging of enlarged $(>1 \mathrm{~cm}$ maximum diameter) mediastinal lymph nodes on CT scan was made by cervical mediastinoscopy. All patients underwent surgical staging with the Mountain revision of the international system for staging lung cancer. ${ }^{2}$ Before the treatment plan was begun, a multidisciplinary team composed of thoracic surgeons, radiation oncologists, pulmonologists, and medical radiologists evaluated all patients.

\section{Treatment Regimen}

Induction protocol. The treatment plan is summarized in Figure E1. Gemcitabine $\left(350 \mathrm{mg} / \mathrm{m}^{2}\right.$, or $300 \mathrm{mg} / \mathrm{m}^{2}$ for patients older than 70 years) was administered weekly as a 30-minute intravenous infusion at least 4 hours before radiation therapy. Concurrent treatment with antiemetics, antibiotics, sedatives, cortisone, hematopoietic growth factors, and gastric protectors was permitted.

Radiotherapy was administered with an individualized approach according to the volume and location of the disease, with the requirement that the $100 \%( \pm 5 \%)$ isodose should encompass the entire target volume, with maximum dose to the spinal cord of 36 Gy. Gross tumor volume was the same as clinical target volume, and planning target volume was clinical target volume plus the surrounding $1.5-\mathrm{cm}$ margin. Elective nodal irradiation was never administered. The prescription dose was $50.4 \mathrm{~Gy}$, administered in daily single $1.8-\mathrm{Gy}$ fractions or in 1.2-Gy doses twice daily on weekdays. CT-based planning was performed in each case. Heterogeneity corrections were applied. A photon beam with 6 to $10 \mathrm{MV}$ energy was used in all cases.

A complete laboratory test with arterial blood gas analysis, together with clinical, oncologic, and pneumologic examinations, was carried out every week before the administration of gemcitabine. A control chest roentgenogram was taken when the dose of 20 to 25 Gy was reached.

In the case of hematologic grade 3 or nonhematologic grade 2 toxicity, the administration of gemcitabine was avoided. Radiotherapy was discontinued if grade 4 hematologic or grade 3 nonhematologic toxicity occurred. Treatment was eventually continued pending toxicity resolution; a maximum 7-day delay was permitted. Any patient with declining performance status or rapid progression of disease during treatment was removed from the protocol.

Clinical response. Four weeks after induction chemoradiotherapy was completed, patients underwent restaging to assess clinical response. Restaging procedures included bronchoscopy, pulmonary function tests, and CT scans of the chest and upper abdomen.

Response to preoperative chemoradiation therapy was evaluated by the multidisciplinary team according to response evaluation criteria in solid tumors. ${ }^{11}$ Complete response was defined as the total disappearance of all radiographic and clinical evidence of disease. Partial response (PR) was defined as any response less than complete but with greater than $30 \%$ reduction in the sum of products of the crossed diameters of all measurable lesions. Patients with less tumor shrinkage were considered to have stable disease (SD). Progressive disease (PD) was defined as a greater than $20 \%$ increase in the sum of the products of the crossed diameters of all measurable lesions or the appearance of new locoregional or metastatic disease. The demonstration of metastatic disease at any time was scored as PD, irrespective of any locoregional improvement. Patients excluded from surgery received multidrug chemotherapy and in selected cases boost irradiation.

Surgical procedure. All patients who at clinical evaluation had a complete response, $\mathrm{PR}$, or SD (given that the resectability criteria had been met) underwent thoracotomy 1 to 2 weeks after restaging. Mediastinoscopy was repeated in select cases. A lateral musclesparing thoracotomy was used for all patients. The goal was to remove the entire tumor area. Resection was considered complete if proximal resection margins were free of tumor on frozen section examination. Lobectomy was preferred when possible; however, a bilobectomy or pneumonectomy was performed for primary tumor invasion or regional lymph node metastases. When necessary, resection of adjacent structures was performed. Unilateral mediastinal lymph node dissection was accomplished with all pulmonary resections. In all patients who underwent resection, the bronchial stump was wrapped in an intercostal pedicled muscle flap.

Toxicity evaluation and feasibility. Adverse effects of induction treatment were recorded according to Common Toxicity Criteria of National Cancer Institute of Canada, version 2.0. Feasibility was assessed by the ability of patients to complete induction therapy, by operability, and by curative resectability (Cancer Therapy Evaluation [http://ctep.cancer.gov/]. Maryland: National Cancer Institute, 1999. Common Toxicity Criteria, version 2.0 [Accessed 2004 May 24]. Available from: http://ctep.cancer. gov/reporting/ctc.html).

Pathologic response. Tumor response was defined as downstaging of $\mathrm{cT}$ or $\mathrm{pN}$ according to the indications of the American Joint Committee on Cancer $^{12}$ : complete pathologic response was defined as ypT0 N0, whereas partial pathologic response was defined as reduction in either $\mathrm{cT}$ or $\mathrm{pN}$ without a reciprocal increase in the other (PD).

Survival. Survival and tumor recurrence were assessed by patient follow-up. Patients were to be followed up monthly for the first 3 months, every 3 months for the next 2 years, every 6 months for the next 3 years, and annually thereafter. Investigations included interim history, physical examination, and laboratory tests. Fiberoptic bronchoscopy and CT of the chest, abdomen, and brain were carried out every 3 months for the first year, every 6 months for the next 3 years, and yearly thereafter, or when clinical signs of recurrence developed. Recurrence was classified as locoregional (inside the ipsilateral thorax, excluding parenchymal nodules, always classified as M), distant (outside the ipsilateral thorax), or both.

\section{Data Analysis}

The survival probabilities were calculated with the Kaplan-Meier method $^{13}$ from the beginning of the induction treatment until death, unavailability for follow-up, or time to evaluation for this report and included patients with operative deaths (defined as occurring within the same hospitalization as the operation or after the initial discharge but related to the operation). Disease-free survival was calculated from the first postoperative day until any event such as tumor recurrence, incidence of second cancer, or secondary condition. Statistical differences between the individual curves were assessed with the log-rank test. The COX proportional hazard model was applied for univariate and multivariate analysis to confirm the prognostic effect of the factors on survival. 
TABLE 1. Patient characteristics ( $n=46$ )

\begin{tabular}{lrr}
\hline Characteristic & No. & $\%$ \\
\hline Age (y) & & \\
Median & 64 & \\
Range & $47-75$ & \\
Sex & & \\
Male & 37 & 80.4 \\
Female & 9 & 19.6 \\
Eastern Cooperative Oncology & & \\
$\quad$ Group performance status & & \\
O & 35 & 76.1 \\
1 & 11 & 23.9 \\
Histologic cell type & & \\
Squamous cell & 24 & 52.1 \\
Adenocarcinoma & 18 & 39.1 \\
Large cell & 2 & 4.4 \\
Adenosquamous & 1 & 2.2 \\
Other & 1 & 2.2 \\
Clinical and TNM staging & & \\
IIIA & & \\
T3 N1 & 41 & 89.1 \\
T1 N2 & 8 & 17.4 \\
T2 N2 & 6 & 13.0 \\
T3 N2 & 12 & 26.1 \\
IIIB & 15 & 32.6 \\
T4 N2 & 5 & 10.9 \\
T1 N3 & 4 & 2.7 \\
\hline
\end{tabular}

\section{Results}

\section{Patient Characteristics}

Between February 2001 and June 2003, a total of 46 patients who met the aforementioned eligibility criteria entered into this phase II trial. Clinical characteristics and staging are given in Table 1 . There were 41 patients with cIIIA disease and 5 with cIIIB. Only 1 enrolled patient had N3 disease confirmed by cervical mediastinoscopy-a small primitive neoplasm involving the contralateral paratracheal nodes. Cervical mediastinoscopy was performed on all patients, with positive results in 39 cases $(84.8 \%)$. Thirty-seven patients $(80.4 \%)$ had ipsilateral mediastinal node involvement (N2 disease): $6 \mathrm{~T} 1,12 \mathrm{~T} 2,15 \mathrm{~T} 3$, and $4 \mathrm{~T} 4$. The last included 2 pulmonary artery invasions and 1 each tracheal and superior vena cava invasions. Clinical N1 stage was documented in 8 patients, all with $\mathrm{T} 3$ tumor.

\section{Induction Therapy}

Thirty-eight of 46 patients undergoing this trial $(82.6 \%)$ received the full-dose induction treatment. During induction therapy, most patients had moderate hematologic toxicity. Only 4 patients required hospitalization: 3 for febrile neutropenia and 1 for anemia necessitating blood transfusion. Moderate nonhematologic toxicity (G1-G2) was seen in 15 patients (32.6\%). In particular, nausea and vomiting were seen in 14 patients (11 G1 and 3 G2), esophageal toxicity was limited to G1 in 5 cases and G2 in 1, and pneumonitis was seen in 2 patients, 1 G1 and 1 G2. No patient had neurotoxicity or nephrotoxicity. There were no toxicity-related deaths from induction therapy.

Five patients ( 4 cIIIA and 1 cIIIB) were not available for restaging: 1 patient underwent surgery for abdominal aortic aneurysm during the treatment, 3 refused to continue treatment, and 1 died of treatment-unrelated causes. Thus a total of 41 patients were available for restaging; of these, 38 patients $(92.7 \%)$ received the full treatment.

No complete clinical responses were recorded. The overall clinical response rate (all PRs) among the patients with restaged disease was $82.9 \%$ (initially 32 cIIIA and 2 cIIIB). Five patients (12.2\%, 4 cIIIA and 1 cIIIB) showed SD, and 2 patients (4.9\%) had PD (initially 1 cIIIA and cIIIB).

No significant correlations were found among response to treatment and clinical characteristics (age, sex, performance status, TNM stage, and histologic type).

\section{Surgery}

Twenty-nine patients (28 with PR and 1 with SD, 27 cIIIA and 2 cIIIB) from the restaged group (70.7\%) who on downstaging reentered the resectability criteria underwent surgery. Of the remaining 12 patients (6 with PR, 4 with SD, and 2 with PD), 4 refused surgery, 5 were judged to have unresectable disease, and 3 were considered medically unable to undergo surgery. Radical resection was possible in all the patients operated on and included lobectomy in 17 patients $(58.6 \%)$, bilobectomy in $4(13.8 \%)$, and pneumonectomy in $8(27.6 \%)$. Overall resectability rate (patients undergoing resection among the entire enrolled population) was $63 \%$. Complex resections included 4 intrapericardial pneumonectomies (13.8\%) and 3 sleeve lobectomies (10.3\%). In most cases, some difficulties related to chemoradiotherapyinduced fibrosis were encountered; nevertheless, a standard major procedure was always performed. Thus the surgical resectability rate (patients undergoing resection among all patients considered appropriate candidates at restaging) was $100 \%$.

No patient died within 30 days after the operation. Postoperative morbidity was $13.8 \%$ (4/29) and included 1 patient with myocardial infarction, 1 with postpneumonectomy empyema, 1 with prolonged air leak, and 1 with lobar atelectasis necessitating twice-daily bronchoscopic aspiration. Overall median postoperative hospitalization was 10 days (range 6-23 days).

\section{Pathologic Response}

Pathologic downstaging to stage 0 or I was observed in 18 patients (62\% of the 29 undergoing resection, or $39 \%$ of the total patient group of 46; Table 2). In particular, there were 5 patients $(17.2 \%)$ who had no evidence of viable tumor cells in the resected specimen (stage 0 , initially 4 cIIIA and 
TABLE 2. Clinical and pathologic downstaging among patients who underwent operation

\begin{tabular}{|c|c|c|c|c|c|c|}
\hline \multirow[b]{2}{*}{ Pathologic staging } & \multicolumn{2}{|c|}{ Stage cIllA } & \multicolumn{2}{|c|}{ Stage clllB } & \multicolumn{2}{|c|}{ Total } \\
\hline & No. & $\%$ & No. & $\%$ & No. & $\%$ \\
\hline 0 & 4 & 13.8 & 1 & 3.4 & 5 & 17.2 \\
\hline IA & 9 & 31.1 & & & 9 & 31.1 \\
\hline IB & 4 & 13.8 & & & 4 & 13.8 \\
\hline IIA & 2 & 6.9 & & & 2 & 6.9 \\
\hline IIB & 2 & 6.9 & & & 2 & 6.9 \\
\hline IIIA & 7 & 24.1 & & & 7 & 24.1 \\
\hline Total & 28 & 96.6 & 1 & 3.4 & 29 & 100 \\
\hline
\end{tabular}

1 cIIIB) and 13 patients with pathologic stage I (9 stage ypIA and 4 ypIB). Of interest, among the 9 patients with downstaging to ypIA, 6 had only microscopic tumor cell foci. Four patients $(13.8 \%)$ had pathologic response to stage II. Thus 22 of 29 patients (75.8\%) responded to preoperative treatment and had their disease downstaged. Three patients (10.3\%) showed a partial pathologic response, including 2 patients with downstaging from cT3 $\mathrm{pN} 2$ to ypT2 ypN2 and 1 patient with downstaging from cT3 pN2 to ypTmic (microscopic tumor remnants) ypN2. In 4 patients (13.8\%), pathologic staging corresponded to the clinical restaging (SD). No pathologic upstaging was observed in the group undergoing operation.

\section{Survival}

Median follow-up was 13 months (range 2-28 months) and was complete for all patients. Median overall survival was 13 months. The calculated 1- and 2-year Kaplan-Meier survivals among all 46 patients were $75 \%$ and $66.1 \%$, respectively (Figure E2). When patients were compared according to resection, the median survival and the 1- and 2-year projected survivals were 19 months, 92.8\%, and $81.8 \%$, respectively, for the operative group of 29 and 5 months, $42.5 \%$, and $36.1 \%$, respectively, for patients who did not undergo operation ( $\mathrm{n}=17, P=.0002$; Figure E3). In the resection group, 25 patients $(86.2 \%)$ were alive at 2 years, with a median disease-free survival of 16 months (Figure E4.) Among the 29 who underwent resection, recurrence developed in 4 (1 local and 3 distant metastases) and was the cause of death in 3 cases. The fourth patient died of cancer-unrelated causes.

\section{Discussion}

Surgery, when possible with radical intent, is the only chance of cure for patients with lung cancer. Good results are obtained with surgery alone in only a few select cases when the disease is diagnosed to be at stage III. The vast majority of patients with stage III NSCLC do not benefit from surgery alone, even when the surgical approach with radical intent is technically feasible. Moreover, patients in this very same class do not benefit either from any therapeutic strategy relying on a single kind of treatment, whether radiotherapy or chemotherapy. In the last two decades, impressive steps forward have been taken in the integration of different treatment modalities in an effort to offer a reasonable chance of cure for this complex disease.

To date, the most frequently adopted treatment strategy for locally advanced NSCLC is concurrent radiochemotherapy. ${ }^{4}$ In very recent years, the discussion has focused on two main points: the eventual induction potential of concurrent radiochemotherapy regimens (demonstrated to be more effective than those of chemotherapy only $)^{5,9,20,21}$ and the value and role of surgery within this setting., ${ }^{4,22}$

An authoritative experience ${ }^{4,22}$ has demonstrated that patients who have been operated on after radiochemotherapy treatment have better results in terms of progression-free survival (median 14 vs 11.7 months, 3 years $29 \%$ vs 19\%). These results have led to a multicenter, randomized, phase III trial (new Intergroup Trial R0333/S0332) in which the radiochemotherapy plus surgery arm is matched with an arm of chemotherapy plus surgery, thus demonstrating a codified role and a solid value of surgery. ${ }^{4,22}$

In the complex clinical environment, as is the case with locally advanced NSCLC, in terms of extreme simplification, we can say that the ideal induction treatment is feasible, safe, and effective, with a negligible impact on surgical morbidity and mortality. The feasibility, safety, and efficacy of induction concurrent radiochemotherapy have been investigated extensively. ${ }^{5,20}$ Frequently, the analysis of pathologic downstaging rate has been used as a surrogate end point in respect to survival. ${ }^{23-25}$

In our personal experience, we have investigated the feasibility and safety of the association of radiotherapy and gemcitabine in a previously reported phase I trial. ${ }^{19}$ In that experience, we demonstrated that concurrent radiochemotherapy treatment with weekly administered gemcitabine (maximum tolerable dose $350 \mathrm{mg} / \mathrm{m}^{2}$ ) plus involved field radiotherapy at the dose of 50.4 Gy was safe and feasible and produced an interesting pathologic downstaging rate in those cases in which, on the basis of favorable clinical response, surgical operation had been incidentally indicated. On the basis of those results, we planned the phase II trial reported in this article.

It is important to highlight the data regarding toxicity, surgical morbidity and mortality, and pathologic downstaging. The treatment has been well tolerated, with a high patient compliance rate (87.7\%). Even though hematologic toxicity was to be expected in the low range, the esophageal and pulmonary toxicity was within low rates relative to similar treatments. ${ }^{26}$ This can be explained by the fact that the adopted radiotherapy volumes were focused on the macroscopic disease with a total dose of $50.4 \mathrm{~Gy}$ (useful for induction approaches but lower than that adopted for radical 
treatments) with the use of 3-dimensional conformal radiotherapy planning.

With regard to surgical morbidity and mortality, despite the fact that it is a generally accepted idea that complication rates could be higher among those patients who are operated on after induction therapy, to date several methodologic differences have biased the interpretation of the reported results. Different kinds of treatment such as induction therapy (chemotherapy only vs radiochemotherapy), different chemotherapeutic agents and schedules, different radiotherapy techniques and schedules, different staging and restaging procedures, and different surgical indication criteria and timing as well as different surgical approaches all render the vast amounts of available data absolutely inhomogeneous. Moreover, and significantly because the most significant expected surgery-related morbidity is pulmonary, functional data and evaluation of pulmonary function at the different stages of any multimodality therapy approach have seldom been reported. ${ }^{27}$ In any case, it is clear that when a pneumonectomy is necessary, the morbidity and mortality correlated with this kind of operation are significantly higher if induction therapy has been administered preoperatively. ${ }^{22,23,28}$

In our experience, surgical morbidity and mortality were within the permitted ranges. This can be explained by the fact that the pneumonectomy rate was low because the extent of operation had been modulated according to the restaged morphologic type of the disease with intraoperative check of surgical radicality. In our opinion, this gives the best value to the downstaging obtained with induction therapy. We retrospectively reviewed the pre-induction therapy clinical data and estimated that 16 of the patients operated $(55.1 \%)$ would actually probably have required a pneumonectomy at presentation, mainly because of central airway involvement (5 patients), bulky hilar nodal disease (10 patients), and vascular involvement (1 patient).

The pathologic downstaging rate to stage 0 or I (18/46, $39.2 \%)$ is similar to that reported by others ${ }^{5,22,29,30}(12.5 \%$ $33.7 \%$ ) and confirms that gemcitabine, even if used at noncytotoxic levels as a single chemotherapeutic agent, is a powerful radioenhancer essentially because a favorable pharmacokinetic characteristic of this drug is the retention of its cytotoxic metabolite within cells, with terminal elimination rates as long as 72 hours. This allows a single shot of gemcitabine to cover more than one radiotherapy application (as many as six in hyperfractionated schedules).

It has been demonstrated that pathologic downstaging in induction therapy trials is directly and significantly correlated with long-term survival, ${ }^{25}$ and our recent experience ${ }^{5}$ confirmed this evidence. The data regarding a positive longterm overall survival trend obtained in this phase II trial in patients with resected disease ( $81.8 \%$ at 2 years) should be considered cautiously in light of the short follow-up range; however, they strengthen the value of pathologic downstaging itself as an effective surrogate end point for the evaluation of any induction therapy protocol efficacy.

In light of the reported experiences, we conclude that induction therapy with gemcitabine and radiotherapy for locally advanced NSCLC is feasible, safe, and effective. It does not impair surgical morbidity and mortality. The obtained rate of pathologic downstaging was reasonably high and was associated with a good long-term survival trend. However, further investigation is encouraged in proper controlled, randomized, phase III trials.

\section{References}

1. American Cancer Society [http://www.cancer.org/]. Cancer Facts \& Figures 2004. Accessed 2004 June 2. Available from: http://www. cancer.org/downloads/STT/CAFF_finalPWSecured.pdf

2. Mountain CF. Revisions in the international system for staging lung cancer. Chest. 1997;111:1710-7.

3. Pfister DG, Johnson DH, Azzoli CG, Sause W, Smith TJ, Baker S Jr, et al. American Society of Clinical Oncology treatment of unresectable non-small-cell lung cancer guideline: update 2003. J Clin Oncol. 2004;22:330-53.

4. Gandara DR,West H, Albain KS, Wasik MW, Crowley JC, Choy H. Defining the role of radiation therapy in combination with preoperative chemotherapy in stage iii non-small cell lung cancer: rationale and design of a new intergroup study. Proc Am Soc Clin Oncol. 2004: 458-62.

5. Trodella L, Granone P, Valente S, Margaritora S, Macis G, Cesario A, et al. Neoadjuvant concurrent radiochemotherapy in locally advanced (IIIA-IIIB) non-small-cell lung cancer: long-term results according to downstaging. Ann Oncol. 2004;15:389-98.

6. Sonett JR, Krasna MJ, Suntharalingam M, Schuetz J, Doyle LA, Lilenbaum M, et al. Safe pulmonary resection after chemotherapy and high-dose thoracic radiation. Ann Thorac Surg. 1999;68:316-20.

7. Machtay M, Lee JH, Stevenson JP, Shrager JB, Algazy KM, Treat J, et al. Two commonly used neoadjuvant chemoradiotherapy regimens for locally advanced stage III non-small cell lung carcinoma: Longterm results and associations with pathologic response. J Thorac Cardiovasc Surg. 2004;127:108-13.

8. Pass HI, Pogrebniak HW, Steinberg SM, Mulshine J, Minna J. Randomized trial of neoadjuvant therapy for lung cancer: interim analysis. Ann Thorac Surg. 1992;53:992-8.

9. Roth JA, Fossella F, Komaki R, Ryan MB, Putnam JB Jr, Lee JS, et al. A randomized trial comparing perioperative chemotherapy and surgery with surgery alone in resectable stage IIIA non-small-cell lung cancer. J Natl Cancer Inst. 1994;86:673-80.

10. Rosell R, Gomez-Codina J, Camps C, Javier Sanchez J, Maestre J, Padilla J, et al. Preresectional chemotherapy in stage IIIA non-smallcell lung cancer: a 7-year assessment of a randomized controlled trial. Lung Cancer. 1999;267:7-14.

11. Pitz CC, Maas KW, Van Swieten HA, de la Riviere AB, Hofman P, Schramel FM. Surgery as part of combined modality treatment in stage IIIB non-small cell lung cancer. Ann Thorac Surg. 2002;74:164-9.

12. Rinaldi M, Crino L. Induction chemotherapy with gemcitabine and cisplatin in stage III non-small cell lung cancer. Lung Cancer. 2001; 34(Supp14):S25-30.

13. Van Zandwijk N, Smit EF, Kramer GW, Schramel F, Gans S, Festen $\mathrm{J}$, et al. Gemcitabine and cisplatin as induction regimen for patients with biopsy-proven stage IIIA N2 non-small-cell lung cancer: a phase II study of the European Organization for Research and Treatment of Cancer Lung Cancer Cooperative Group (EORTC 08955). J Clin Oncol. 2000;18:2658-64.

14. Aapro MS, Martin C, Hatty S. Gemcitabine-a safety overview. Anticancer Drugs. 1998;9:191-201.

15. Shewach DS, Lawrence TS. Radiosensitization of human solid tumor cell lines with gemcitabine. Semin Oncol. 1996;23(5 Suppl 10):65-71. 
16. Pacini S, Milano F, Pinzani P, Pazzagli M, Gulisano M, Ruggiero M, et al. Effects of gemcitabine in normal and transformed human lung cell cultures: cytotoxicity and increase in radiation sensitivity. Tumori. 1999;85:503-7.

17. Lawrence TS, Eisbruch A, McGinn CJ, Fields MT, Shewach DS Radiosensitization by gemcitabine. Oncology (Williston Park). 1999; 13(10 Suppl 5):55-60.

18. Argiris A, Liptay M, LaCombe M, Marymont M, Kies MS, Sundaresan S, et al. A phase I/II trial of induction chemotherapy with carboplatin and gemcitabine followed by concurrent vinorelbine and paclitaxel with chest radiation in patients with stage III non-small cell lung cancer. Lung Cancer. 2004;45:243-53.

19. Trodella L, Granone P, Valente S, Turriziani A, Macis G, Corbo GM, et al. Phase I trial of weekly gemcitabine and concurrent radiotherapy in patients with inoperable non-small-cell lung cancer. J Clin Oncol. 2002;20:804-10.

20. Albain KS, Rusch VW, Crowley JJ, Rice TW, Turrisi AT 3rd, Weick $\mathrm{JK}$, et al. Concurrent cisplatin/etoposide plus chest radiotherapy followed by surgery for stages IIIA (N2) and IIIB non-small-cell lung cancer: mature results of Southwest Oncology Group phase II study 8805. J Clin Oncol. 1995;13:1880-92.

21. Sugarbaker DJ, Herndon J, Kohman LJ, Krasna MJ, Green MR. Results of cancer and leukemia group B protocol 8935. A multiinstitutional phase II trimodality trial for stage IIIA (N2) non-small-cell lung cancer. Cancer and Leukemia Group B Thoracic Surgery Group. J Thorac Cardiovasc Surg. 1995;109:473-83.

22. Albain KS, Scott CB, Rusch VR, Turrisi AT, Shepherd FA, Smith C, et al for RTOG, SWOG, NCIC, ECOG, CALGB, NCCTG. Phase III comparison of concurrent chemotherapy plus radiotherapy (CT/RT) and CT/RT followed by surgical resection for stage IIIA (pN2) nonsmall cell lung cancer (NSCLC): initial results from intergroup trial 0139 (RTOG 93-09) [abstract 2497]. Proc Am Soc Clin Oncol. 2003;22:621.

23. Martin J, Ginsberg RJ, Venkatraman ES, Bains MS, Downey RJ, Korst $\mathrm{RJ}$, et al. Long-term results of combined-modality therapy in resectable non-small-cell lung cancer. J Clin Oncol. 2002;20:1989-95.

24. Betticher DC, Hsu Schmitz SF, Totsch M, Hansen E, Joss C, von Briel $\mathrm{C}$, et al. Mediastinal lymph node clearance after docetaxel-cisplatin neoadjuvant chemotherapy is prognostic of survival in patients with stage IIIA pN2 non-small-cell lung cancer: a multicenter phase II trial. $J$ Clin Oncol. 2003;21:1752-9.

25. Choi NC, Carey RW, Daly W, Mathisen D, Wain J, Wright C, et al. Potential impact on survival of improved tumor downstaging and resection rate by preoperative twice-daily radiation and concurrent chemotherapy in stage IIIA non-small-cell lung cancer. J Clin Oncol. 1997; 15:712-22.

26. Gandara DR, Chansky K, Albain KS, Leigh BR, Gaspar LE, Lara PN $\mathrm{Jr}$, et al. Consolidation docetaxel after concurrent chemoradiotherapy in stage IIIB non-small-cell lung cancer: phase II Southwest Oncology Group Study S9504. J Clin Oncol. 2003;21:2004-10.

27. Granone P, Cesario A, Margaritora S, Galetta D, Valente S, Corbo GM, et al. Morbidity after induction therapy and surgery in non small cell lung cancer (NSCLC). Focus on pulmonary function. Lung Cancer. 2002;36:219-20.

28. Doddoli C, Thomas P, Thirion X, Seree Y, Giudicelli R, Fuentes P. Post-operative complications in relation with induction therapy for lung cancer. Eur J Cardiothorac Surg. 2001;20:385-90.

29. Grunenwald DH, Andre F, Le Pechoux C, Girard P, Lamer C, Laplanche A, et al. Benefit of surgery after chemoradiotherapy in stage IIIB (T4 and/or N3) non-small cell lung cancer. J Thorac Cardiovasc Surg. 2001;122:796-802.

30. Strauss GM, Herndon JE, Sherman DD, Mathisen DJ, Carey RW, Choi $\mathrm{NC}$, et al. Neoadjuvant chemotherapy and radiotherapy followed by surgery in stage IIIA non-small-cell carcinoma of the lung: report of a Cancer and Leukemia Group B phase II study. J Clin Oncol. 1992;10:1237-44.

\section{Discussion}

Dr Ross M. Bremner (Los Angeles, Calif). Galetta and colleagues are to be congratulated on an excellent presentation and for per- forming a well-designed single-institution study. The article is also well written and thoughtful. This group has used a combination of a radiosensitizing drug, gemcitabine, at low dose with concurrent radiotherapy in a neoadjuvant setting in an attempt to improve the survival results of patients with this difficult stage III NSCLC. This is a phase II study, and an interim analysis at that, and we should keep that in mind in our interpretation of the results. The surgical results are impressive, with no deaths and low morbidity, although 1 postpneumonectomy empyema may have been related to a bronchial stump complication. The 2-year survival among the resection group is also impressive, although caution should be exercised when interpreting the comparison between the survival of patients who underwent resection and those who did not. These are two very different populations and probably represent different tumor biologic characteristics. In fact, I think that these groups should probably not be compared. Most of the patients with unresected disease were medically unfit for surgery or had progression of disease on induction therapy, obviously constituting a group with a worse prognosis. Nonetheless the early results of those patients who underwent resection do appear promising and warrant further study. The pathologic response rates are also impressive. These results may in part be related to the sensitizing effect of gemcitabine but are also probably related to the conformal approach of radiotherapy. This 3-dimensional approach has altered the radiation therapist's ability to focus higher doses of radiation to more precisely defined fields with lessening of side effects to surrounding tissue, and I think the results of radiotherapy today are different from those of a decade ago, as the literature is beginning to attest. This approach with low-dose gemcitabine combined with radiotherapy is really designed at improving local control, and it appears to be effective in that. The gemcitabine was too low a dose to have a significant systemic effect. I was interested to note that most patients had squamous cell carcinoma, which is more frequently a local problem than either adenocarcinoma or large cell cancer. In many countries, America included, the overwhelming majority of NSCLCs now are adenocarcinomas, and I think we all subscribe to the paradigm that N2 disease should be considered systemic disease, even if not apparent on our current imaging modalities. To this end, perhaps there is greater value to neoadjuvant chemotherapy with systemic cytotoxic doses before addressing local disease with radiotherapy and surgery.

I have two questions, Dr Galetta. First, among the recurrences seen in your series, how many of them were adenocarcinoma, and where were the sites of the recurrence? Second, there is no mention in the article of adjuvant treatment in these patients. Were any treated with postoperative chemotherapy? If not, will you change your practice in light of the recent data, some from your country, that suggest a benefit of chemotherapy for this group of patients undergoing resection?

Dr Cesario. Dr Bremner, thank you very much for your comments and questions. Regarding the first question, we had 4 recurrences. Three were distant, and 1 was local. Distant recurrences were all in patients with adenocarcinoma and all at the level of the brain.

Regarding your second question, this was a trial that was designed before the International Adjuvant Lung Cancer Trial (IALT) results and before Adjuvant Lung Cancer Project Italy (ALPI) which was the study made in my country regarding adju- 
vant chemotherapy. So adjuvant chemotherapy has been indicated on the basis of pathologic staging. No adjuvant chemotherapy was administered for pathological stage I but it was given for pathological stage II and III. In highly selected cases, boost radiation therapy was given.

Dr Robert J. Cerfolio (Birmingham, Ala). That was excellent work. We appreciate you bringing the interim analysis to us; it is helpful. I have a few questions. First, you didn't mention your policy or general belief about restaging and surgical selection. Do you believe that recalcitrant N2 disease-according to repeated mediastinoscopy, repeated endoscopic ultrasonography with fineneedle aspiration, or even thoracotomy with frozen section-is a contraindication to resection? If results are positive, are there any patients for whom you still resect?

Dr Cesario. We have discussed the matter of remediastinoscopy in a recent paper published during the last year, and we do not believe that remediastinoscopy can be of any help in N2 cases. It can be of help in N3 cases, but in our experience with remediastinoscopy, all the operations were performed by the same senior surgeon who did the first mediastinoscopy. He encountered several difficulties in performing the operation, and once he reached the area of biopsy and of rebiopsy, he was not certain to have resampled the same area. When we sent frozen sections, the pathologist was saying we were crazy if we wanted a diagnosis on that tissue. So remediastinoscopy is quite a difficult issue in our opinion.

Dr Cerfolio. Do you operate if there is recalcitrant N2 disease, then?

Dr Cesario. Just on CT scan evidence.

Dr Cerfolio. So you don't use tissue confirmation. My second question concerns bronchial buttressing. I assume you are using an intercostal muscle flap harvested before chest retraction or a serratus. Do you use these? My final question concerns the use of a $60-\mathrm{Gy}$ dose before the operation rather than a 45-Gy dose. We have come to prefer a higher, curative dose, because if after restaging patients still have N2-positive disease and surgery is denied, they do not have a large gap in the completion of their "curative dose" of radiotherapy, which would hinder its efficacy. We have also done resections in more than 75 patients after 60-Gy radiotherapy, and we find the operation and postoperative complications no different. Do you have any comments on this strategy, or any experience in pulmonary resection after 60-Gy therapy?

Dr Cesario. Your question was about protection of the bronchial stump?

Dr Cerfolio. Right.

Dr Cesario. Yes. Not 60 Gy but 50 Gy.
Dr Cerfolio. Have you operated after $60 \mathrm{~Gy}$ ?

Dr Cesario. No, no, 50.4 Gy.

Dr Cerfolio. I understand that for this series, but what about in other cases?

Dr Cesario. Every patient had the bronchial stump protected by intercostal muscle flap.

Dr Douglas Wood (Seattle, Wash). I have two brief questions. First, were you doing mediastinoscopy on every patient, so that these are all mediastinoscopy-proven IIIA disease, or was this predominantly based on radiologic staging? Second, regarding the extent of resection; were these all R0 resections? You did not mention whether you had any R1 or R2 resections.

Dr Cesario. To your first question, we did mediastinoscopy in all cases in which the CT scan led us to suspect mediastinal involvement, so all patients with clinical N2 disease underwent mediastinoscopy.

To your second question, yes, we had all R0 resection. To maximize the effect of the induction treatment, we check the resection intraoperatively for completeness in an organ-sparing attitude. This is intended to give the maximum strength to the effect of the chemotherapy. In a T4 case, for example a big tumor invading the pulmonary artery, if you have a good response so that you can do a lobectomy rather than a pneumonectomy, we find that this is the correct way, but intraoperatively you have to check whether resection is complete.

Dr Wood. Well, it's impressive to have all R0 resections in this kind of series, so I congratulate you. Finally, what is the perceived purpose or benefit of this regimen? Are you trying to decrease toxicity or improve outcomes relative to standard therapy with cisplatin-based combination chemoradiotherapy? Are you trying to make it so that every patient can get through it more easily, or do you think it will actually result in better outcomes?

Dr Cesario. Thank you for this question, because it provides an occasion for me to explain what we are doing now. We were trying to find a good local treatment for patients with bad local disease. What we are doing now for patients with T4 stage IIIB disease is to give them systemic chemotherapy, three cycles of full-dose chemotherapy, and then restage. If we find that they have a response, we can give them this kind of local treatment. Coming after systemic chemotherapy at full doses, it should be less toxic. This is what we are doing now. Our aim was to find a local treatment that was both effective and less toxic to be given after systemic chemotherapy at full dosage in a complex multimodality three-stage treatment: chemotherapy, induction radiochemotherapy, and then, if possible, surgery. 


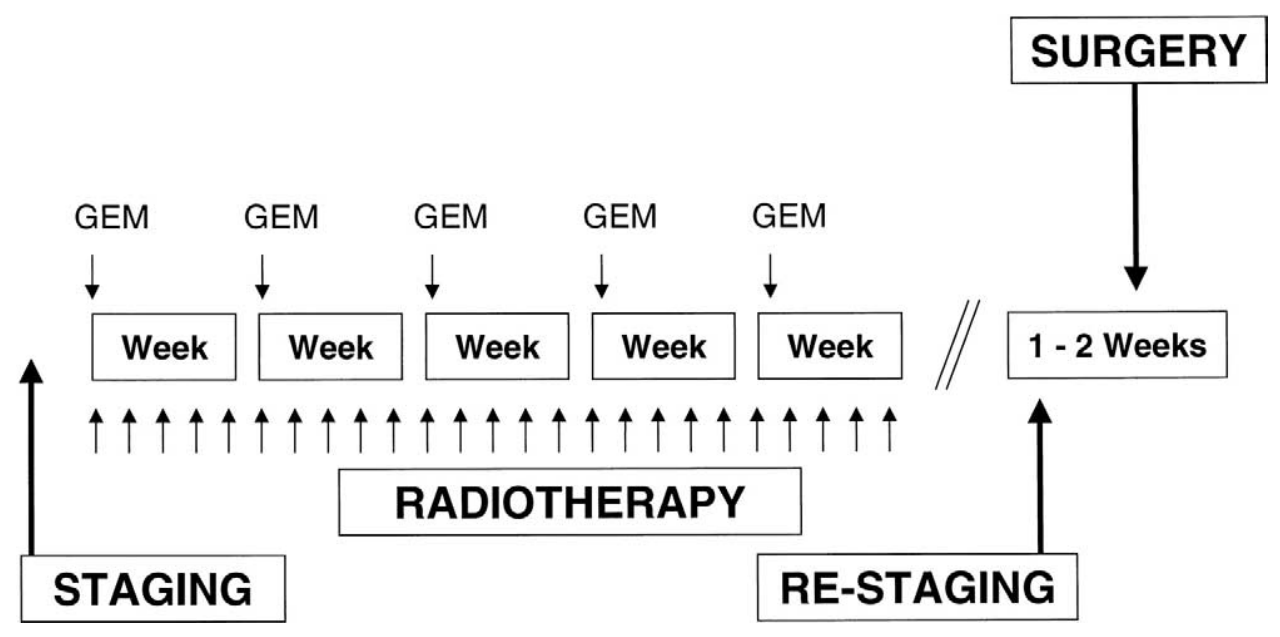

Figure E1. Treatment plan. GEM, Gemcitabine. 


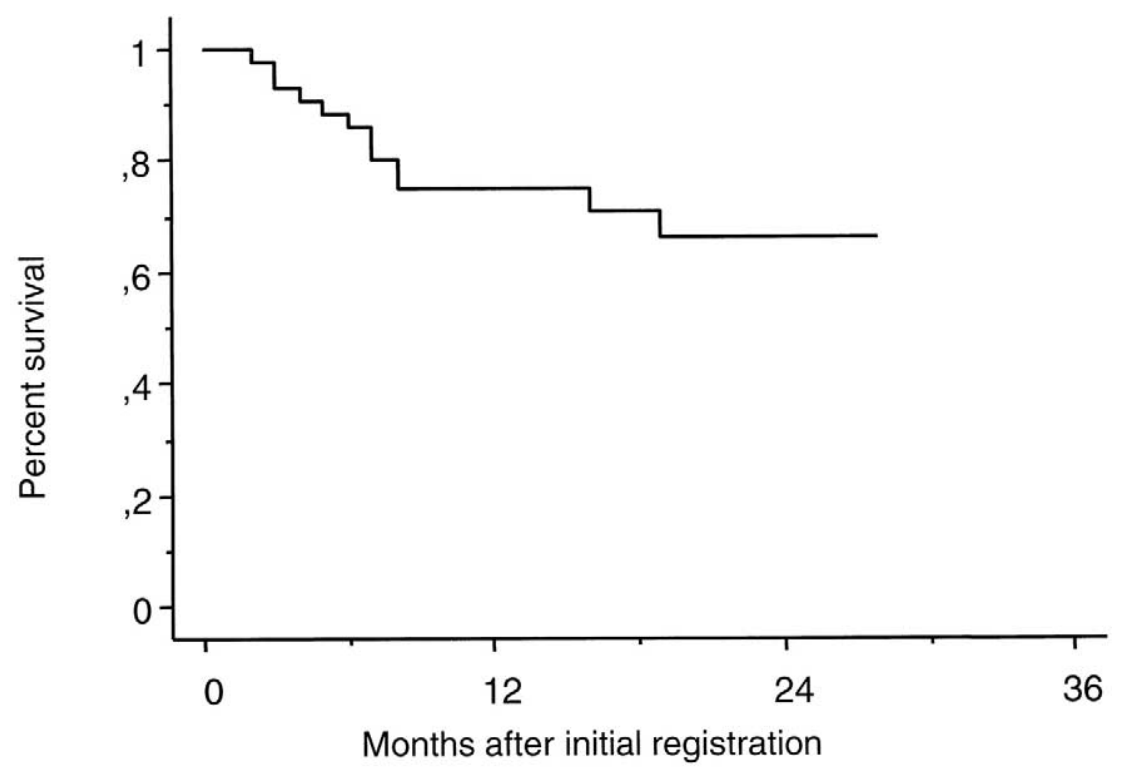

No. at risk

Figure E2. Survival curve of all eligible patients. Numbers at bottom indicate patients at risk. 


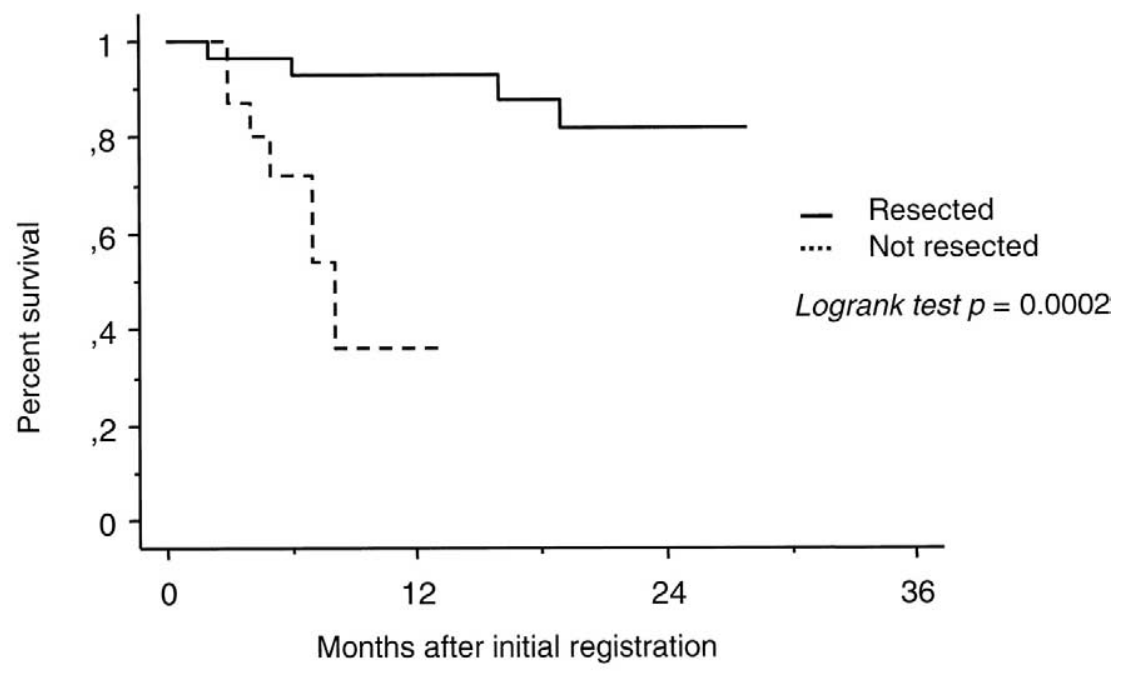

No. at risk

$\begin{array}{lrrr}R & 29 & 27 & 25 \\ \text { NR } & 17 & 9 & \end{array}$

Figure E3. Survival curves for patients with resected ( $R$, solid line) versus nonresected (NR, dotted line) disease. Numbers at bottom indicate patients at risk.

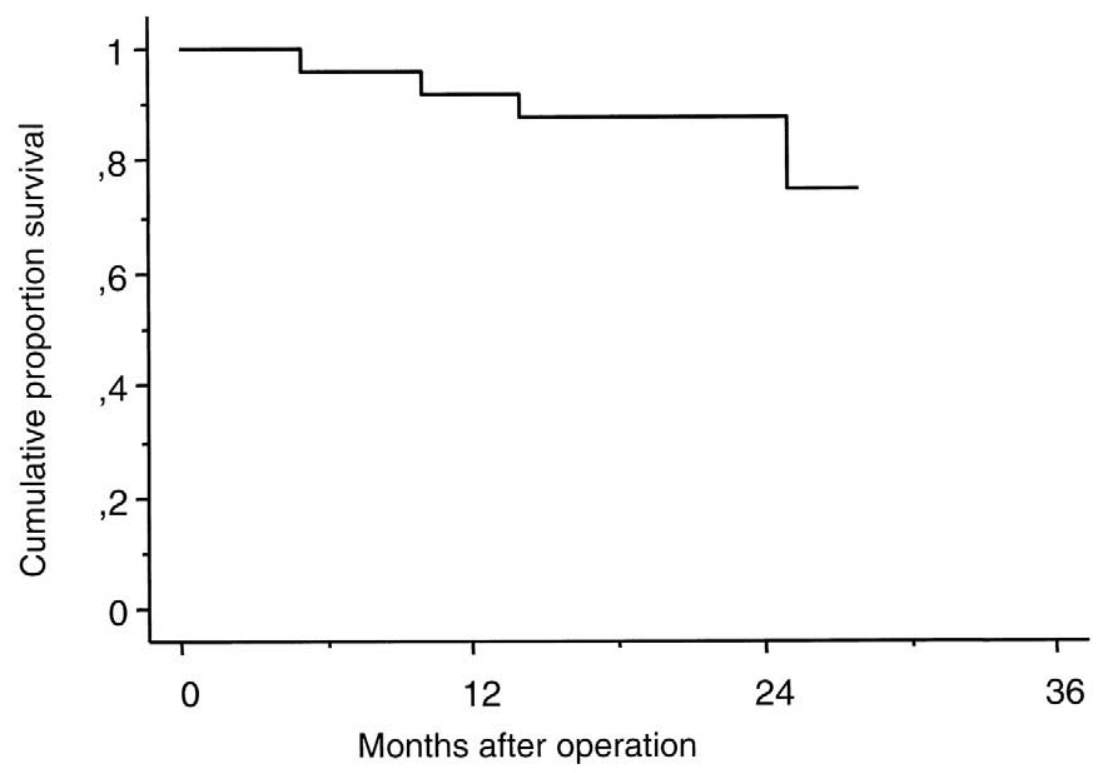

No. at risk

29

27

26

Figure E4. Disease-free survival among patients undergoing resection. Numbers at bottom indicate patients at risk. 